\title{
BMJ Open Effect of vitamin D replacement on maternal and neonatal outcomes: a randomised controlled trial in pregnant women with hypovitaminosis D. A protocol
}

\author{
M Chakhtoura, ${ }^{1}$ A Nassar, ${ }^{2}$ A Arabi, ${ }^{1}$ C Cooper, ${ }^{3} \mathrm{~N}$ Harvey, ${ }^{3}$ Z Mahfoud, ${ }^{4}$ \\ M Nabulsi, ${ }^{5}$ G El-Hajj Fuleihan ${ }^{1}$
}

To cite: Chakhtoura $M$, Nassar A, Arabi A, et al. Effect of vitamin $D$ replacement on maternal and neonatal outcomes: a randomised controlled trial in pregnant women with hypovitaminosis $D$. A protocol. BMJ Open 2016;6: 010818.

doi:10.1136/bmjopen-2015010818

- Prepublication history and additional material is available. To view please visit the journal (http://dx.doi.org/ 10.1136/bmjopen-2015010818).

Received 10 December 2015 Revised 20 January 2016 Accepted 3 February 2016

CrossMark

For numbered affiliations see end of article.

Correspondence to Dr M Chakhtoura; mc39@aub.edu.lb

\section{ABSTRACT}

Introduction: The vitamin $\mathrm{D}$ recommended doses during pregnancy differ between societies. The WHO guidelines do not recommend routine prenatal supplementation, but they underscore the fact that women with the lowest levels may benefit most. The effects of routine supplementation during pregnancy on maternal and neonatal clinical outcomes have not been investigated in the Middle East, where hypovitaminosis $D$ is prevalent. Our hypothesis is that in Middle Eastern pregnant women, a vitamin D dose of $3000 \mathrm{IU} /$ day is required to reach a desirable maternal 25-hydroxyvitamin $D[25(\mathrm{OH}) \mathrm{D}]$ level, and to positively impact infant bone mineral content (BMC).

Methods and analysis: This is a multicentre blinded randomised controlled trial. Pregnant women presenting to the Obstetrics and Gynaecology clinics will be approached. Eligible women will be randomised to daily equivalent doses of cholecalciferol, $600 \mathrm{IU}$ or $3000 \mathrm{IU}$, from 15 to 18 weeks gestation until delivery. Maternal 25(OH)D and chemistries will be assessed at study entry, during the third trimester and at delivery. Neonatal anthropometric variables and 25(OH)D level will be measured at birth, and bone and fat mass assessment by dual-energy $\mathrm{X}$-ray absorptiometry scan at 1 month. A sample size of 280 pregnant women is needed to demonstrate a statistically significant difference in the proportion of women reaching a 25 $(\mathrm{OH}) \mathrm{D}$ level $\geq 50 \mathrm{nmol} / \mathrm{L}$ at delivery, and a difference in infant BMC of $6(10) \mathrm{g}$, for a $90 \%$ power and a $2.5 \%$ level of significance. The proportions of women achieving a target $25(\mathrm{OH}) \mathrm{D}$ level will be compared between the two arms, using $\chi^{2}$. An independent $t$ test will be used to compare mean infant BMC between the two arms. The primary analysis is an intention-to-treat analysis of unadjusted results.

Ethics and dissemination: The protocol has been approved by the Institutional Review Board at the American University of Beirut-Lebanon (IM.GEHF.22). The trial results will be published in peer-reviewed medical journals and presented at scientific conferences. Trial registration number: NCT02434380.
Strengths and limitations of this study

- This is the first randomised controlled trial (RCT) in the Middle East directly addressing the applicability of the Institute of Medicine recommendations in this specific population, and assessing the effect of vitamin D supplementation on neonatal bone mineral content.

- Multiple maternal and neonatal outcomes that have not been targeted in any previous trial in pregnancy will be assessed as secondary or exploratory outcomes; indeed, the results will guide future research projects in this field.

- Vitamin D and other calciotropic hormones will be measured using the gold standard method, liquid chromatography-tandem mass spectrometry (LC-MS/MS).

- The findings of this trial will help guide the public health policymaker regarding vitamin $D$ supplementation in pregnant women and will allow a step forward in evidence-based recommendations specific to the Middle East.

- Results derived from nutrient RCT, specifically vitamin $D$ supplementation, suffer from several confounding factors related to the baseline status, intake of other nutrients, such as calcium and proteins, and sun exposure, which are difficult to quantify accurately.

\section{INTRODUCTION}

\section{Vitamin D physiology during pregnancy}

Pregnancy is characterised by physiological changes in mineral metabolism, to allow calcium accretion in the fetal skeleton. ${ }^{1-3}$ These changes start in the first trimester, and culminate during the third trimester, a period during which fetal calcium requirements increase exponentially. ${ }^{2}$ Indeed, it is in anticipation of such requirements that maternal calcitriol levels increase during 
pregnancy. While the total calcitriol levels double in the first trimester, free calcitriol levels do not increase until the third trimester and remain so until lactation. ${ }^{2} 4$ Conversely, parathyroid hormone (PTH) levels decrease early on and increase back to mid-normal range by term. ${ }^{2}$ The total calcium level decreases during pregnancy, due to haemodilution, while the ionised calcium level remains stable. ${ }^{2}$ Vitamin D binding proteins also increase during pregnancy secondary to high oestrogen levels,${ }^{45}$ but the 25-hydroxyvitamin $\mathrm{D}(25(\mathrm{OH}) \mathrm{D})$ level, the single best nutritional indicator of vitamin D status, ${ }^{6}$ remains stable. ${ }^{7}$ The changes in calcitriol levels led to the description of pregnancy as a state of 'absorptive hypercalciuria'. ${ }^{12}$ The above adaptive physiology is key to safety considerations when using vitamin D supplementation during pregnancy, as well as to determining key biochemical and hormonal parameters to be monitored.

\section{Maternal vitamin D status during pregnancy}

Vitamin D deficiency during pregnancy is prevalent worldwide, especially in developing countries. ${ }^{8}$ In a systematic review of 18 studies conducted in Western countries during the first trimester, white Caucasian pregnant women were found to have a mean $25(\mathrm{OH}) \mathrm{D}$ level between 29 and $73 \mathrm{nmol} / \mathrm{L} .{ }^{9}$ Mean $25(\mathrm{OH}) \mathrm{D}$ levels were lower in non-Caucasian pregnant women, ranging between 15.2 and $43 \mathrm{nmol} / \mathrm{L} .{ }^{9}$ In addition to ethnicity, higher latitude was a significant predisposing factor for hypovitaminosis D. ${ }^{9}$ Similarly, in non-Western countries, more than half of the pregnant women who were beyond their first trimester had 25(OH)D levels below $75 \mathrm{nmol} /$ $\mathrm{L}$; these include countries such as India, ${ }^{10}$ Kuwait, ${ }^{11}$ Pakistan ${ }^{12}$ and Turkey. ${ }^{13}$ Even lower levels $(<25 \mathrm{nmol} / \mathrm{L})$ have been reported at delivery in Saudi Arabia, Iran and the United Arab Emirates. ${ }^{14}$ Furthermore, immigrant women were at particular risk. ${ }^{15}{ }^{16}$ An observational study from the Netherlands showed significantly lower $25(\mathrm{OH})$ $\mathrm{D}$ levels during the first trimester in immigrant pregnant women (Turkish, Moroccan and others), compared to western participants. ${ }^{17}$

\section{Association between maternal vitamin D status and maternal adverse outcomes}

Vitamin D insufficiency during pregnancy is associated with adverse maternal outcomes such as increased risk of gestational diabetes mellitus (GDM), preeclampsia, caesarean-section delivery and bacterial vaginosis. ${ }^{18}$ In a recent meta-analysis of observational studies, the risk of GDM was found to be increased by $40-84 \%$ in pregnant women with low $25(\mathrm{OH}) \mathrm{D}$ levels, defined as $<50 \mathrm{nmol} / \mathrm{L}$ or $<75 \mathrm{nmol} / \mathrm{L}$, depending on the studies. ${ }^{19-21}$ While preeclampsia risk was significantly increased in vitamin D insufficient women, ${ }^{22}$ C-section rates were inconsistently affected by vitamin D status. ${ }^{23}$ However, these findings remain limited by the inherent biases of observational studies, inconsistent adjustment for confounders, in addition to the wide heterogeneity in vitamin $\mathrm{D}$ assays and vitamin $\mathrm{D}$ cut-offs definition.
Association between maternal vitamin D level and neonatal adverse outcomes

Low maternal $25(\mathrm{OH}) \mathrm{D}$ levels were recently linked to fetal programming, and were found to be associated with adverse events in neonates, resulting in small for gestational age (SGA) at birth, ${ }^{19}{ }^{23}$ and also later on during childhood, leading to reduced muscle and bone mass in offspring at 4 and 9 years. ${ }^{24}{ }^{25}$ This may be explained by the fact that maternal vitamin $\mathrm{D}$ is essential for fetal musculoskeletal integrity, as it regulates neonatal bone accrual, possibly through specific proteins that are responsible for placental calcium transport. ${ }^{26}$ Recently, data from the Southampton Women's Survey (SWS) showed that maternal 25(OH)D level is significantly correlated with placental amino acid transporters expression, that mediate the transport of various nutrients to the fetus. ${ }^{27}$ Furthermore, maternal vitamin D may influence the fetal muscle motor unit size, and consequently muscle mass and strength after birth. ${ }^{25}$ It is noteworthy that fetal bone development is one of the predictors of peak bone mass, adult bone mineral content and hip geometry, thus correlating with fracture risk later in life. ${ }^{26} 28$

\section{Vitamin D replacement guidelines during pregnancy}

The guidelines regarding vitamin D replacement or supplementation during pregnancy vary substantially.

The 2010 Institute Of Medicine (IOM) Report on Dietary Reference Intakes for Calcium and Vitamin D recommended $600 \mathrm{IU}$ to pregnant women as the recommended daily allowance (RDA), the RDA being the dose that is projected to allow at least $97.5 \%$ of the pregnant women population to reach the desirable target $25(\mathrm{OH})$ D level $\geq 50 \mathrm{nmol} / \mathrm{L}^{29}$ This recommendation was based on observational studies, none of which were conducted in the Middle East. ${ }^{29}$ Conversely, the Endocrine Society 2011 guidelines recommended that 1500-2000 IU daily of vitamin $\mathrm{D}$ is needed to reach a target $25(\mathrm{OH}) \mathrm{D}$ level $\geq 75 \mathrm{nmol} / \mathrm{L}$ (a recommendation that was graded as weak with moderate quality of evidence). ${ }^{30}$ The American College of Obstetricians and Gynecologists (ACOG) does not recommend screening for vitamin D level in pregnancy, and abides by the IOM recommendations. ${ }^{31}$ Moreover, the WHO 2012 guidelines on vitamin $\mathrm{D}$ replacement during pregnancy did not recommend vitamin D supplementation as part of prenatal care. ${ }^{32}$ This was based on a meta-analysis of vitamin D trials during pregnancy, which identified a limited number of high-quality studies demonstrating a beneficial effect of supplementation on maternal and neonatal outcomes, and concluded that further randomised controlled trials (RCTs) are needed..$^{33}$ In the UK, however, pregnant women are considered at risk of vitamin D deficiency, and supplementation with $400 \mathrm{IU}$ daily is required. ${ }^{34}$

It is not clear whether any of the above recommended doses are applicable to non-western populations, with lower baseline vitamin D levels, such as in Lebanon and other Middle Eastern countries. Indeed, the WHO 
pregnancy guidelines clearly stated that "Vitamin D supplementation will probably have the most benefit in populations of poor countries, those with darker skin colour and in populations with a high prevalence of vitamin $\mathrm{D}$ deficiency. It is expected that this intervention would be acceptable to women who are not exposed to adequate amounts of sunshine." 32 This is particularly relevant to our population that tends to avoid sunshine, wear concealed clothing or use sunblock, all resulting in the low 25(OH)D levels observed across the life cycle.

\section{RCTs of vitamin D supplementation during pregnancy}

Two landmark RCTs have been conducted in the USA ${ }^{35}$ and the UK. ${ }^{36}$ Hollis et $a l^{35}$ showed that in pregnant women in the USA, with a baseline $25(\mathrm{OH}) \mathrm{D}$ level around $60 \mathrm{nmol} / \mathrm{L}$, a vitamin dose of $4000 \mathrm{IU}$ daily allowed $82 \%$ of participants to reach a $25(\mathrm{OH}) \mathrm{D}$ level of $80 \mathrm{nmol} / \mathrm{L}$, while only $70 \%$ and $50 \%$ reached this target in the intermediate (2000 IU daily) and low (400 IU daily) doses, respectively. Cooper et $a l^{36}$ showed that vitamin D supplementation of 1000 IU daily, compared to placebo, in pregnant women in the UK allowed a significant increase in Bone Mineral Content (BMC) of neonates, however, only when they were born in winter. One study from India, comparing non-intervention to vitamin $\mathrm{D}$ supplementation groups, with the dose being dependent on $25(\mathrm{OH}) \mathrm{D}$ levels at 20 weeks gestation, showed that vitamin $\mathrm{D}$ supplementation resulted in a significant difference in the achieved $25(\mathrm{OH}) \mathrm{D}$ level at delivery $(43.1(81.3) \mathrm{nmol} / \mathrm{L}$ in the former group versus $56.8(47.5) \mathrm{nmol} / \mathrm{L}$ in the latter group) ${ }^{37}$ Hollis $e t a l^{36}$ and Sablok $e t a \hat{l}^{37}$ showed that vitamin D supplementation decreased the risk of preterm labour, gestational diabetes and hypertensive complications (all combined).

In the Middle East and North Africa region, there are few recent RCTs that attempted to determine the optimal regimen of vitamin D replacement in healthy pregnant women. ${ }^{38-41}$ With the exception of Soheilykhah $e t a l^{40}$ who assessed the effect of vitamin D supplementation on insulin resistance, the primary outcomes in these studies were mostly maternal and neonatal 25(OH)D levels (see online supplementary appendix 1). None of the other clinically important outcomes, such as neonatal size and other anthropometric measurements, neonatal BMC, GDM and C-section rates, were evaluated as primary outcomes in any of these trials (see online supplementary appendix 1).

We therefore compiled a registry of all ongoing vitamin $\mathrm{D}$ trials in pregnancy, as captured by their registration on clinicaltrial.gov ${ }^{42}$ (see online supplementary appendix 2). In these trials, different doses of vitamin $\mathrm{D}$, reaching up to $7000 \mathrm{IU}$ daily, are being administered, and the outcomes to be assessed include neonatal weight and length, childhood asthma, maternal bone mineral density, maternal adverse outcomes, including preeclampsia and preterm labour, and neonatal adverse outcomes, such as SGA. Only two of the ongoing trials are being conducted in the Middle East, one in Iran and one in Israel, start supplementation in the third trimester and use vitamin D doses of $7000 \mathrm{IU}$ and $2000 \mathrm{IU}$ daily, respectively. These latter studies aim at assessing the effect of vitamin D supplementation on offspring calcium status, maternal and infant vitamin D status and bone status (by quantitative ultrasound) at 1 year. Neither addresses the applicability of IOM vitamin D dose recommendations in pregnant women in the Middle East. Three completed (unpublished) trials were identified (see online supplementary appendix 2), two from the USA and one from Pakistan. They assessed the effect of various doses of vitamin $\mathrm{D}$ on the $25(\mathrm{OH}) \mathrm{D}$ level, immune function and periodontal disease.

\section{Hypothesis}

The study hypothesis is that a high dose of vitamin $\mathrm{D}$, equivalent to $3000 \mathrm{IU} /$ day, is needed to optimise maternal vitamin $\mathrm{D}$ level and neonatal musculoskeletal parameters, compared to a low dose of $600 \mathrm{IU} /$ day.

\section{Objectives}

The two primary objectives of this trial, comparing the effect of high-dose versus low-dose vitamin $\mathrm{D}$, are as follows:

- The proportion of women who will reach the IOM defined desirable $25(\mathrm{OH}) \mathrm{D}$ level $\geq 50 \mathrm{nmol} / \mathrm{L}$ at delivery.

- Infant BMC at 1 month.

The secondary objectives are to compare the effect of high-dose versus low-dose vitamin D on:

- Maternal outcomes:

- Mean maternal 25(OH)D level at delivery.

- Mean maternal PTH level at delivery.

- Mean change in maternal urine calcium.

- Neonatal outcomes:

- Mean neonatal 25(OH)D level at delivery.

- Mean neonatal PTH level at delivery.

- Mean neonatal fat and lean mass at 1 month.

- Mean neonatal knee to heel length at birth.

Exploratory outcomes include a composite outcome (incidence of GDM and C-section), maternal weight, blood pressure, ill days, fetal and neonatal anthropometric measures, including neonatal length and weight, rate of small for gestational age, APGAR score, placental weight and $1 \alpha$-hydroxylase activity, in addition to other placental and genetic studies, that characterise mineral and fuel metabolism.

\section{METHODS AND ANALYSIS}

The protocol of this trial was developed on the basis of Standard Protocol Items: Recommendations for Interventional Trials (SPIRIT); see online supplementary appendices $3-6$ for further details. This protocol is registered on clinicaltrial.gov (NCT 02434380, April 2015). 


\section{Study design}

This study is a phase III multicentre blinded randomised controlled superiority trial with two arms, conducted at the American University of Beirut-Medical Center (AUB-MC), Rafic Hariri University Hospital (RHUH) and Bahman Hospital.

\section{Recruitment}

Pregnant women in their first trimester will be recruited from the obstetric private clinics and outpatient departments of the three participating centres (AUB-MC, RHUH and Bahman Hospital). Information about the trial will be available as Arabic and English flyers in the obstetrics and gynaecology department, as well as the private clinics and outpatient departments of the three centres. The flow chart of participants and details of study visits are summarised in figure 1 .

\section{Randomisation}

The allocation sequence will be a computer-generated, permuted block randomisation, stratified by study centre, with a 1:1 allocation. The statistician will be responsible for sequence generation and treatment assignment. The senior pharmacist at AUB-MC will be responsible for treatment allocation.

\section{Concealment and blinding}

Vitamin D and placebo pills are manufactured to have a similar shape, colour, size, smell and taste. The study medications will be stored at the AUB-MC pharmacy, and placebo and/or vitamin $\mathrm{D}$ pills will be dispensed in boxes. Boxes will be sequentially numbered as per the random allocation list by the pharmacist. The pharmacist keeps the list linking the randomisation code to the participant identity/trial number and to the delivered box number. At each visit, the pharmacist allocates a box to every participant, containing enough pills until the next visit, with dates at which pills should be administered. The research assistant collects the boxes in sealed envelopes prior to each participant's visit and delivers them at the end of the visit.

The research assistants, the healthcare providers, the principal investigator, the co-investigators and the biostatistician do not have access to code break, and are all blinded to the treatment allocation. The only personnel who will not be blinded will be the pharmacist.

\section{Investigational medicinal product}

All participants receive once per week two tablets that are similar in shape, colour, size, smell and taste. Each tablet can be either a placebo or a $10000 \mathrm{IU}$ vitamin D (cholecalciferol), provided by Europharm.

The high-dose group receives two tablets of $10000 \mathrm{IU}$ weekly (equivalent daily dose 2857 IU).

The low-dose group receives one tablet of $10000 \mathrm{IU}$ and one tablet of placebo alternating with two tablets of placebo on a weekly basis (equivalent daily dose 714 IU).
Vitamin D supplementation present in prenatal multivitamins will be permitted up to 200 IU daily, which will raise the aforementioned treatment doses to approximately $3050 \mathrm{IU}$ and $900 \mathrm{IU}$ daily, respectively.

The manufacturers had no role in the study design or implementation.

\section{Study visits}

\section{A. Prescreening visit}

Trained research assistants will approach pregnant women who are in their first trimester during their routine prenatal visits to study sites. Eligible pregnant women willing to participate and to be compliant with the study protocol, and who provide written informed consent, will be invited to a screening visit.

B. Screening visit

The screening visit will be scheduled to coincide with the nuchal translucency appointment date (between 11 and 13 weeks of gestation). During this visit, eligibility criteria will be verified and blood tests for $25(\mathrm{OH}) \mathrm{D}$ level, calcium, phosphate, magnesium, creatinine and thyroid stimulating hormone (TSH) will be withdrawn. Urine calcium will be assessed in a fasting urine spot or $24 \mathrm{~h}$ urine collection (table 1$)$. The level of $25(\mathrm{OH}) \mathrm{D}$ will be measured using the electrochemiluminescence immunoassay (ECLIA) at the AUB-MC Clinical Chemistry laboratory. Reference ranges using this assay are defined as follows: Deficiency $<25 \mathrm{nmol} / \mathrm{L}$, insufficiency $25-62.4 \mathrm{nmol} / \mathrm{L}$, desirable $>62 \mathrm{nmol} / \mathrm{L}$, toxic $>374 \mathrm{nmol} / \mathrm{L}$. AUB-MC clinical chemistry laboratory partakes in the quality assurance, evaluation and accreditation by the College of American Pathologists ${ }^{43}$ and is a participant in the Vitamin D External Quality Assurance Surveillance (DEQAS) programme. ${ }^{44}$

\section{Eligibility criteria}

Inclusion criteria:

- Pregnant women gestational age $(\mathrm{GA})<14$ weeks at screening visit. ${ }^{\mathrm{i}}$

- Middle Eastern origin; Middle East countries as defined by the World Bank (Bahrain, Egypt, Iran, Iraq, Palestine, Jordan, Kuwait, Lebanon, Oman, Qatar, Saudi Arabia, Syria, the United Arab Emirates, Yemen). ${ }^{45}$

- $25(\mathrm{OH}) \mathrm{D}$ level between 25 and $75 \mathrm{nmol} / \mathrm{L}$.

- Age $>18$ years.

Vitamin D supplementation $\leq 200$ IU daily.

Exclusion criteria:

in the case where the pregnant woman presents after 13 weeks GA,
she is still eligible for the screening visit provided that screening blood
tests are carried out before 16.5 weeks of gestation and the first visit in
the trial occurs before 18 weeks GA.
ii If daily vitamin D supplementation is between 200 and 600 IU daily, at
enrollment, the pregnant women will be advised to adjust prenatal
multivitamin doses, in consultation with her primary obstetrician, to
ensure that total vitamin D supplementation during the study does not
exceed $1400 \mathrm{IU} /$ week, in consultation with the primary obstetrician. 


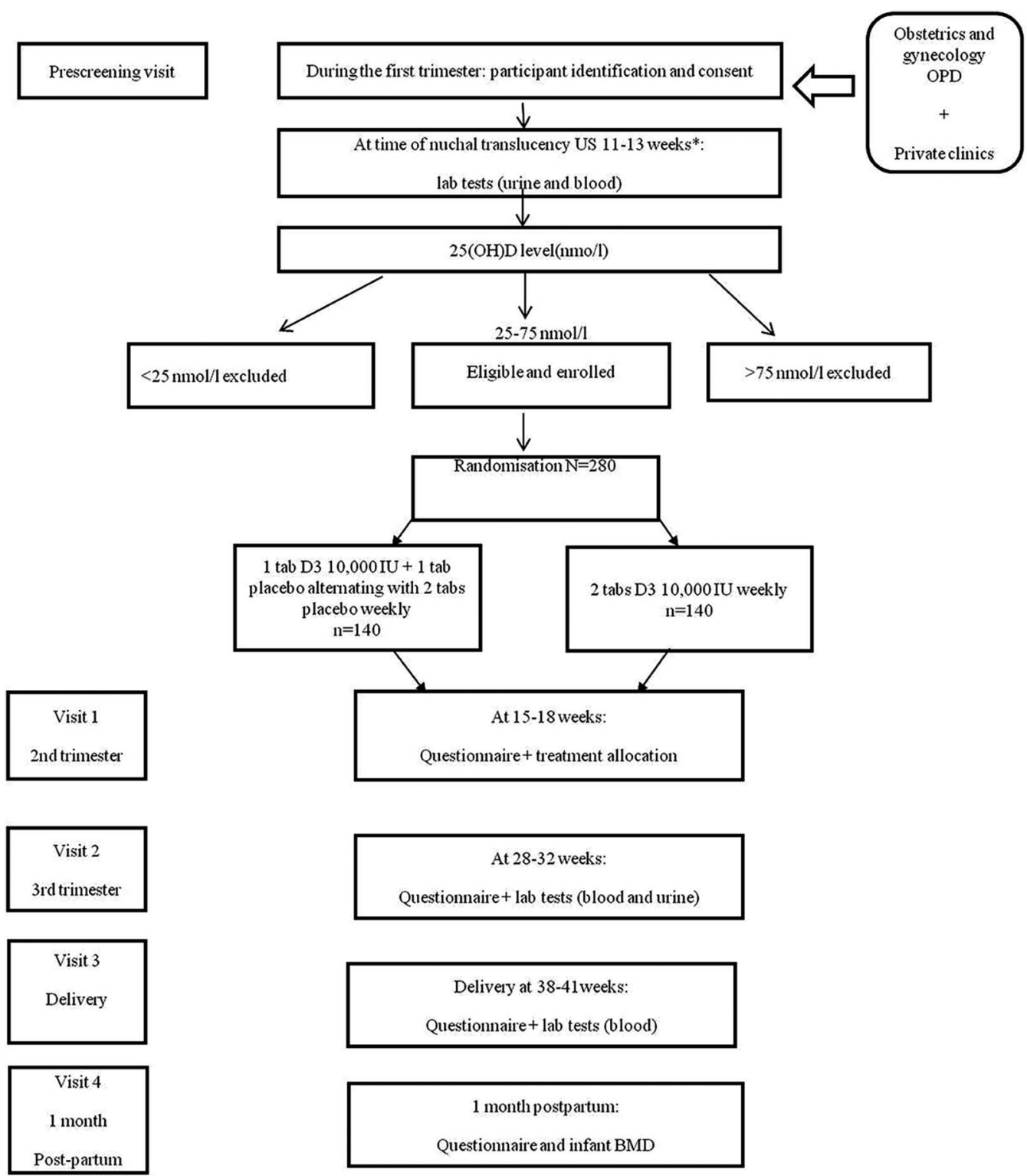

*In case the pregnant woman presents after 13 weeks of gestation, she is still eligible for screening visit provided that screening blood tests are done before 17.5 weeks and the first $y$ isit in the trial occurs before 18 weeks of gestation.

Figure 1 Trial flow chart. BMD, bone mineral density.

- $25(\mathrm{OH}) \mathrm{D}$ level $<25 \mathrm{nmol} / \mathrm{L}$, as it would be unethical to randomise pregnant women to the low dose of vitamin $\mathrm{D}$, and $25(\mathrm{OH}) \mathrm{D}$ level $>75 \mathrm{nmol} / \mathrm{L}(30 \mathrm{ng} /$ $\mathrm{mL}$ ), as vitamin $\mathrm{D}$ supplementation with routine prenatal multivitamins would be sufficient.

- Known metabolic bone disease or chronic diseases associated with bone abnormalities (renal or liver diseases.

- Current medications likely to interfere with vitamin D metabolism (enzyme inducing anticonvulsants, antituberculosis).

- Vitamin D supplementation >600 IU daily. ${ }^{\text {iii }}$

\footnotetext{
iii* If a pregnant woman is on a high dose of vitamin D supplementation, > $600 \mathrm{IU}$ daily, vitamin D should be stopped at least 1 month prior to study entry, at the discretion of her primary physician.
}

Fetal physical anomalies on the initial ultrasound.

- Renal stones.

- Hyperparathyroidism.

- Uncontrolled thyroid dysfunction.

- Diagnosis of cancer in the past 10 years (other than basal cell carcinoma).

- Serum calcium $>10 \mathrm{mg} / \mathrm{dL}$.

- Diabetes mellitus type 1 or type 2 .

- Previous GDM.

- Allergy to any component of vitamin D formulation.

C. First visit

During the first visit at $15-18$ weeks GA, a questionnaire will be administered to collect maternal information on parity, demographics, smoking and alcohol history, exercise, previous medical problems, medications, dietary calcium and vitamin $\mathrm{D}$ intake, in addition to relevant paternal information. Pregnant women will 
Table 1 Trial events and outcomes measures

\section{1-13 weeks}

(screening

Trial event and outcomes measures

visit)

15-18 weeks (visit 1)

Maternal height

Maternal weight, blood pressure

Maternal health and diet assessment

Maternal 25(OH)D, Crea, Ca

Maternal cell count, ph, mg, alb, TSH, $1,25(\mathrm{OH})_{2} \mathrm{D}$

PTH

Maternal glucose $(1 \mathrm{~h})$

Maternal $24 \mathrm{~h}$ (or spot) urine collection for calcium and

creatinine

Maternal vitamin D binding protein

Maternal genetic pathways of vitamin D metabolism

Fetal US:

crown -rump

Fetal US:

- Femur length

- Abdominal circumference

- Head circumference

- Biparietal diameter

Newborn weight, length, knee to heel length APGAR score

Placental weight

Placental studies

Newborn 25(OH)D, Ca, PTH (cord blood)

Newborn genetic pathways of vitamin $\mathrm{D}$ metabolism

Infant bone/fat mass

Infant weight and length

Infant health and diet assessment

$\sqrt{ }$ Test performed for clinical purpose.

$\times$ Test performed for research purpose.

PTH, parathyroid hormone; TSH, thyroid-stimulating hormone; US, ultrasound 
be randomised early in their second trimester to one of two vitamin $\mathrm{D}$ doses as discussed above.

\section{Second visit}

This visit will take place at 28-32 weeks GA, during which maternal weight and blood pressure will be recorded, maternal health and diet assessed, in addition to assessment of adverse events, if any. We will check compliance to trial medication by pill counting. Blood and urine tests will be carried out (see table 1).

E. Third visit

The third visit will coincide with the participant's delivery. When entering labour, the research team will be informed about each participant by the obstetrician, or by the participant, or her partner. The research assistant will visit the participant on the first day post partum, and will record information on delivery mode, delivery course and complications, if any. In addition, neonatal measurements at birth such as length, weight and knee -heel length will be recorded in triplicate. Knee-heel length will be measured using handheld vernier calipers. ${ }^{46}$ Knee-heel length measurement is operator dependent; hence, measurements will be carried out in triplicate, and only by paediatricians/neonatologists who are trained on how to use such instruments.

The neonatal 25(OH)D level will be obtained from cord blood, whereas maternal blood tests will be withdrawn when the pregnant woman presents in labour. In addition, blood tests at delivery include genetic studies such as vitamin $\mathrm{D}$ genes polymorphism and RNA expression of vitamin D polymorphisms. After delivery, placental sampling will be performed by a trained medical doctor and samples will be preserved and stored at $-80^{\circ} \mathrm{C}$.

F. Fourth visit

This visit will occur when the infant is 1 month of age. He will undergo bone mineral density (BMD) assessment by dual-energy X-ray absorptiometry (DXA) scan, Hologic machine, Horizon A, V.13.5.3.1, at AUBMC. Infant DXA assessment is performed by technicians certified by the International Society for Clinical Densitometry (ISCD). The technician positions the laser light so that it is centred about $2 \mathrm{~cm}$ below the iliac crest (or umbilicus/belly button) on the child, and observes the emerging image to ensure that the spine is centrally positioned and straight, and that the top of the iliac crests and all of L5 are visible.

In addition, during this visit, information about the infant's health and feeding will be recorded using an interviewer-led questionnaire.

\section{Sample size calculation and justification}

Sample size was calculated for the two primary outcomes: the proportion of pregnant women who will reach a $25(\mathrm{OH}) \mathrm{D} \geq 50 \mathrm{nmol} / \mathrm{L}$ at delivery, and the infant BMC at 1 month; the largest number was considered the final sample size. Given that we have two primary outcomes, type I error was considered $2.5 \%{ }^{47}$ Sample size calculation was performed online. ${ }^{48}$
Sample size calculation for the proportion of women who will reach a $25(\mathrm{OH}) \mathrm{D} \geq 50 \mathrm{nmol} / \mathrm{L}$ at delivery

On the basis of a retrospective lab study conducted at AUB-MC in 2014, the median $25(\mathrm{OH}) \mathrm{D}$ level in the Lebanese population was found to be $52 \mathrm{nmol} / \mathrm{L}$ $(20.9 \mathrm{ng} / \mathrm{mL})$. The low-dose group will receive $10000 \mathrm{IU}$ vitamin D weekly, equivalent to $700 \mathrm{IU}$ daily. The highdose group will receive $20000 \mathrm{IU}$ vitamin D weekly, equivalent to $2850 \mathrm{IU}$ daily. Considering that each 100 IU vitamin D supplementation increases the level by $1.7 \mathrm{nmol} / \mathrm{L},{ }^{49}$ the expected levels reached in the low-dose and high-dose arms would be $67 \mathrm{nmol} / \mathrm{L}$ and $106 \mathrm{nmol} / \mathrm{L}$, respectively. This computation takes into consideration that all groups will be taking additional 200 IU vitamin D daily from their prenatal vitamin pills, thus increasing the final vitamin D intake approximately to $900 \mathrm{IU} /$ day in the low-dose group and $3050 \mathrm{IU} /$ day in the high-dose group. The expected proportions of pregnant women who would reach a $25(\mathrm{OH}) \mathrm{D}$ level $\geq 50 \mathrm{nmol} / \mathrm{L}$, using a SD of $24.9 \mathrm{nmol} / \mathrm{L}$, and assuming normality, would be $75 \%$ and $98.4 \%$, in the low-dose and high-dose arms, respectively. To detect statistical significance between the two groups, for a $90 \%$ power and a type I error of 2.5\%, 50 participants per arm are needed.

Calculation was also carried out on the basis of the results of a recently completed systematic review and meta-analysis of RCTs from the Middle East and North Africa (MENA), conducted by Chakhtoura et al, as part of a Master of Sciences in Health Research thesis project (available online from the Jafet Library at the American University of Beirut-Lebanon). This meta-analysis showed that, in pregnant women from the MENA region, a vitamin D dose of 800-2000 IU daily results in an increase in the $25(\mathrm{OH}) \mathrm{D}$ level by $2.5 \mathrm{nmol} / \mathrm{L}$, and a high dose of $>2000$ IU daily results in an increase in the $25(\mathrm{OH}) \mathrm{D}$ level by $1.67 \mathrm{nmol} / \mathrm{L}$. Starting from a baseline $25(\mathrm{OH}) \mathrm{D}$ level of $52 \mathrm{nmol} / \mathrm{L}$, the $25(\mathrm{OH}) \mathrm{D}$ levels achieved would be $74.5 \mathrm{nmol} / \mathrm{L}$ and $103 \mathrm{nmol} / \mathrm{L}$ in the low-dose and highdose groups, respectively. Accordingly, $83.6 \%$ and $98.3 \%$ would reach the target $25(\mathrm{OH}) \mathrm{D}$ level of $50 \mathrm{nmol} / \mathrm{L}$, and 72 participants per arm are needed for an $80 \%$ power and a type I error of $2.5 \%$. It is noteworthy that the studies included in the aforementioned meta-analysis had a baseline $25(\mathrm{OH}) \mathrm{D}$ level of $20-27 \mathrm{nmol} / \mathrm{L}$, lower than the expected levels in our participants.

\section{Sample size calculation for infant BMC}

Estimations were based on the preliminary results of the MAVIDOS trial conducted by our collaborators at Southampton University, UK. They showed a significant difference of $6 \mathrm{~g}(\mathrm{SD} 10 \mathrm{~g})$ in neonatal mean BMC in the vitamin D supplemented group, compared to placebo, in the winter season. ${ }^{36}$ For a $90 \%$ power and a type I error of $2.5 \%$, considering an SD of BMC of $10 \mathrm{~g}$, to detect a $6 \mathrm{~g}$ difference in BMC between high-dose and low-dose groups, 69 participants per arm are needed. Taking into consideration that $25(\mathrm{OH}) \mathrm{D}$ levels in RHUH and Bahman hospital are lower compared to 
pregnant women presenting to AUB-MC and to pregnant women in the $\mathrm{UK}$, a significant improvement in $\mathrm{BMC}$ is expected throughout the year in the high-dose group compared to the low-dose group.

The largest sample size of 69 participants per arm is our target. If we consider a $50 \%$ dropout rate, to be conservative, approximately 140 participants per arm should be recruited for a total of 280 pregnant women for the whole study. If we consider that $50 \%$ of pregnant women presenting to clinics are eligible, approximately a total of 560 pregnant women should be screened initially. If $30 \%$ of pregnant women accept to participate in clinical trials, approximately 1870 pregnant women should be approached initially.

\section{Statistical analysis}

Baseline demographic characteristics will be summarised using frequencies and percentages for categorical characteristics, and mean $\pm \mathrm{SD}$ (or median and range) for continuous variables. Normality of all variables will be checked. Comparisons between dose groups will be performed using $\chi^{2}$ tests for categorical variables, and $\mathrm{t}$ test for continuous variables, as appropriate.

\section{Unadjusted analysis}

Two primary outcomes are considered:

A. The proportion of women who reach a $25(\mathrm{OH}) \mathrm{D}$ $\geq 50 \mathrm{nmol} / \mathrm{L}$ : binary outcome; the percent of women achieving $25(\mathrm{OH}) \mathrm{D} \geq 50 \mathrm{nmol} / \mathrm{L}$ in the low dose will be compared to those in the high dose using $\chi^{2}$, by constructing a $95 \%$ CI for the difference and computing an unadjusted RR and its 95\% CI, along with the $p$ value. A number needed to treat (NNT) will also be computed.

B. The mean infant BMC at 1 month: continuous outcome; an independent $\mathrm{t}$ test will be used to compare mean BMC between the two arms. 95\% CI for the difference will be calculated.

Secondary and exploratory outcomes:

For secondary and exploratory outcomes, a t test will be used for continuous outcomes and $\chi^{2}$ will be used for binary outcomes to compare means and proportions, respectively. Non-parametric tests including the Wilcoxon sign rank test and Fisher's exact test will be used, respectively, instead of t test and $\chi^{2}$, when needed.

Relative Risk (RR) with corresponding CIs will be calculated for dichotomous variables, and difference in means with their $95 \%$ CIs will be used for additional analysis of continuous variables.

The primary analysis is an intention-to-treat analysis (ITT) of unadjusted results, ITT being defined as the analysis of all participants as randomised, regardless of whether they respected the study protocol or not (effectiveness). The $p$ values will be reported to four decimal places.

For the primary outcomes, $\mathrm{p}$ values will be considered statistically significant if $\leq 0.025$.

SPSS V.23 will be used to conduct statistical analysis.
In case of missing data, analysis restricted to results of individuals with complete data will be carried out (with retrospective power calculation) and compared to analysis resulting from multiple imputations to try to test the robustness of results. ${ }^{50}$

\section{Additional analysis}

Subgroup analysis

As discussed earlier, the IOM targets a $25(\mathrm{OH}) \mathrm{D}$ level of $\geq 50 \mathrm{nmol} / \mathrm{L}^{29}$ and the Endocrine Society targets a level of $\geq 75 \mathrm{nmol} / \mathrm{L}$. ${ }^{30}$ Subgroup analysis based on a $25(\mathrm{OH})$ D level at study entry $(<50 \mathrm{nmol} / \mathrm{L}$ vs $\geq 50 \mathrm{nmol} / \mathrm{L})$ will be carried out to explore whether the treatment effects persist across all $25(\mathrm{OH}) \mathrm{D}$ categories, whether below or above $50 \mathrm{nmol} / \mathrm{L}$.

Subgroup analysis based on the season will be also performed to check for interaction between the vitamin D dose and the season of pregnant women enrolment.

\section{Sensitivity analysis}

Sensitivity analysis will be performed, including Per Protocol analysis and as treated analysis. In addition, an adjusted analysis will be performed, including adjustment for variables that are not evenly distributed between the two arms, if any, and adjustment for variables that are clinically important (even if there is no imbalance in the baseline characteristics of the 2 groups); this includes the baseline 25(OH)D level, pre-pregnancy body mass index, season at enrolment and smoking status.

\section{Ethical considerations}

We will restrict enrolment to pregnant women whose 25 $(\mathrm{OH}) \mathrm{D}$ levels range between 25 and $75 \mathrm{nmol} / \mathrm{L}$. This is because it will be unethical to include women with levels $<25 \mathrm{nmol} / \mathrm{L}$ in the trial, as there is a risk to randomly allocate them to the low-dose arm. In addition, women with a $25(\mathrm{OH}) \mathrm{D}$ level $>75 \mathrm{nmol} / \mathrm{L}$ will be excluded in order to prevent reaching supranormal levels of $25(\mathrm{OH})$ $\mathrm{D}$ should they be allocated to the high-vitamin $\mathrm{D}$ dose. It is noteworthy that high doses of vitamin D (up to 4000 IU daily) have been used in previous trials conducted during pregnancy with no reported adverse events (see online supplementary appendix 1).

The infant radiation exposure resulting from the study procedure, BMD testing by DXA, is minimal. The radiation dose is estimated at $0.007 \mathrm{mSv}$ for whole body DXA. This dose is equivalent to $20 \mathrm{~h}$ of exposure to background radiation, based on the Duke Radiation Safety online assessment and statement. ${ }^{51}$

\section{Safety considerations}

Information on adverse events will be regularly collected soon after starting the trial intervention and during each trial visit. In between visits, all participants will be called by the research team every 2 weeks to emphasise compliance with treatment regimens and to enquire about adverse events. All information will be documented in case report forms and discussed with the Trial 
Monitoring Committee (TMC) (see online supplementary appendix 3 for further details). The TMC will report any serious adverse event to IRB and the Data Safety and Monitoring Board (DSMB) within $48 \mathrm{~h}$.

\section{Dissemination}

Trial results will be communicated to participants, to the public, and to healthcare professionals at AUB-MC and in Lebanon. Results will be presented in scientific meetings and conferences and published in peer-reviewed medical journals, whether the results are in the expected direction or not.

\section{DISCUSSION}

Hypovitaminosis D is a well-recognised common public health problem in Lebanon and in most countries of the Middle East. Many observational studies suggest that maternal hypovitaminosis $\mathrm{D}$ is associated with adverse maternal and neonatal outcomes. Vitamin D RCTs in pregnancy are scarce, with small sample sizes, and their primary outcomes are mostly limited to measuring 25 $(\mathrm{OH}) \mathrm{D}$ levels in mothers and neonates. Furthermore, given the lack of evidence-based guidelines that define the optimal RDA for vitamin D supplementation during pregnancy in our population, and the limited number of randomised clinical trials completed so far in our region, this trial will fill an important knowledge gap. We will conduct this RCT to test the impact of two different doses of vitamin $\mathrm{D}$ replacement on clinically relevant maternal and neonatal outcomes in Middle Eastern women. The Lebanese and other Middle Eastern women in the reproductive age are ideally suited for such trials, in view of the fact that the median $25(\mathrm{OH}) \mathrm{D}$ levels in this age group is relatively low. These levels are reflective of the median low levels registered in most countries from the Middle East, as well as those from Northern Africa. ${ }^{14}$ The doses used will allow us to directly address the applicability of the IOM in our region. The findings of this trial will help guide the public health policymaker regarding vitamin D supplementation in pregnant women and will allow a step forward in evidence-based recommendations specific to the Middle East. Multiple outcomes that have never been targeted in any previous trial in pregnancy will be assessed as secondary or exploratory outcomes; indeed, the results will guide future research projects in this field.

Findings from our trial, and similar to results derived from nutrient RCTs, are prone to the confounding effect of several factors. ${ }^{52}$ Indeed, the baseline $25(\mathrm{OH}) \mathrm{D}$ level, the dietary intake of vitamin $\mathrm{D}$ and other nutrients, such as calcium and proteins, sun exposure and others, remain important predictors affecting the response to vitamin D supplementation, but are very difficult to quantify accurately.

\section{Trial status}

The study was launched on 27 July 2015.
Author affiliations

${ }^{1}$ Calcium Metabolism and Osteoporosis Program, Department of Internal Medicine, American University of Beirut, Beirut, Lebanon

${ }^{2}$ Department of Obstetrics and Gynecology, American University of Beirut, Beirut, Lebanon

${ }^{3} \mathrm{MRC}$ Lifecourse Epidemiology Unit, University of Southampton,

Southampton, UK

${ }^{4}$ Department of Public Health, Weill Cornell Medical College, Doha, Qatar

${ }^{5}$ Department of Pediatrics and Adolescent Medicine, American University of Beirut, Beirut, Lebanon

Acknowledgements This work was made possible thanks to the Scholars in HeAlth Research Program (SHARP). The investigators would like to thank the following: Dr Elie Akl for his assistance in the development of the trial protocol; Members of the data safety and monitoring board: Dr Christopher Gallagher, Dr Munro Peacock, Dr Michael Holick; Dr Munro Peacock for his input on urinary calcium measurements; Professor Heidi Kalkwarf for sharing the guidance on performing lumbar spine scans in infants; Professor James Ware for his input on the trial protocol; Dr Steve Balian and Professor Robert Habib for developing the online database forms; Trial coordinator: Maya Rahmeh; DXA technicians at AUB-MC: Samia Mroueh and Jinane Kronfol. Europharm for providing the vitamin $D$ and placebo tablets for the trial.

Contributors GEHF conceived the idea of the trial. MC, GEHF and AN designed and developed the study protocol. ZM provided advice on the sample size, study design and randomisation. CC and NH provided advice on the trial design, end points, conduct and operating procedures. AA and MN provided advice on the logistic planning of the study. All authors made significant contributions to the protocol development. They all reviewed the draft versions and approved the final version of this manuscript.

Funding The trial has received funding from the Medical Resource Plan at the American University of Beirut, Lebanon, and from the WHO/EMRO under the EMRPPH grant scheme, project number EMIER1409518. Marlene Chakhtoura's work is supported in part by funds from the Scholars in HeAlth Research (SHARP), NIH Grant D43TW009118.

Competing interests Professor Cyrus Cooper reports having received consultancy and honoraria from Alliance for Better Bone Health, Amgen, Eli Lilly, GSK, Medtronic, Merck, Novartis, Pfizer, Roche, Servier, Takeda and UCB, outside the submitted work. All other authors report no conflicts of interest.

Ethics approval This protocol has been approved by the Institutional review Board (IRB) at AUB-MC and RHUH and by the medical committee at Bahman Hospital (Protocol number IM.GEHF 22).

Provenance and peer review Not commissioned; externally peer reviewed.

Open Access This is an Open Access article distributed in accordance with the Creative Commons Attribution Non Commercial (CC BY-NC 4.0) license, which permits others to distribute, remix, adapt, build upon this work noncommercially, and license their derivative works on different terms, provided the original work is properly cited and the use is non-commercial. See: http:// creativecommons.org/licenses/by-nc/4.0/

\section{REFERENCES}

1. Kovacs CS, Fuleihan Gel-H. Calcium and bone disorders during pregnancy and lactation. Endocrinol Metab Clin North Am 2006;35:21-51.

2. Kovacs CS. Calcium and bone metabolism disorders during pregnancy and lactation. Endocrinol Metab Clin North Am 2011;40:795-826.

3. Parkes I, Schenker JG, Shufaro Y. Parathyroid and calcium metabolism disorders during pregnancy. Gynecol Endocrinol 2013;29:515-19.

4. Bouillon $\mathrm{R}$, Van Assche FA, Van Baelen $\mathrm{H}$, et al. Influence of the vitamin D-binding protein on the serum concentration of 1 , 25-dihydroxyvitamin D3: significance of the free 1 25-dihydroxyvitamin D3 concentration. J Clin Invest 1981;67:589.

5. Van Hoof HJ, de Sévaux RG, van Baelen $\mathrm{H}$, et al. Relationship between free and total 1, 25-dihydroxyvitamin $D$ in conditions of modified binding. Eur J Endocrinol 2001;144:391-6.

6. Zerwekh JE. Blood biomarkers of vitamin D status. Am J Clin Nutr 2008;87:1087S-91S. 
7. Kovacs CS. Maternal vitamin D deficiency: fetal and neonatal implications. Semin Fetal Neonatal Med 2013;18:129-35.

8. Arabi A, El Rassi R, El-Hajj Fuleihan G. Hypovitaminosis D in developing countries-prevalence, risk factors and outcomes. Nat Rev Endocrinol 2010;6:550-61.

9. Nassar N, Halligan GH, Roberts CL, et al. Systematic review of first-trimester vitamin $D$ normative levels and outcomes of pregnancy. Am J Obstet Gynecol 2011;205:208.e1-7.

10. Sahu M, Bhatia V, Aggarwal A, et al. Vitamin D deficiency in rural girls and pregnant women despite abundant sunshine in northern India. Clin Endocrinol 2009;70:680-4.

11. Molla AM, Al Badawi M, Hammoud MS, et al. Vitamin D status of mothers and their neonates in Kuwait. Pediatr Int 2005;47:649-52.

12. Hossain N, Khanani R, Hussain-Kanani F, et al. High prevalence of vitamin D deficiency in Pakistani mothers and their newborns. Int J Gynaecol Obstet 2011;112:229-33.

13. Ergür AT, Berberoğlu M, Atasay $B$, et al. Vitamin D deficiency in Turkish mothers and their neonates and in women of reproductive age. J Clin Res Pediatr Endocrinol 2009;1:266.

14. Bassil D, Rahme M, Hoteit M, et al. Hypovitaminosis D in the Middle East and North Africa: prevalence, risk factors and impact on outcomes. Dermatoendocrinol 2013;5:274.

15. Lips P. Worldwide status of vitamin D nutrition. J Steroid Biochem Mol Biol 2010;121:297-300.

16. Van Der Meer IM, Middelkoop BJ, Boeke AJ, et al. Prevalence of vitamin $\mathrm{D}$ deficiency among Turkish, Moroccan, Indian and sub-Sahara African populations in Europe and their countries of origin: an overview. Osteoporos Int 2011;22:1009-21.

17. Van Der Meer IM, Karamali NS, Boeke AJ, et al. High prevalence of vitamin $D$ deficiency in pregnant non-Western women in The Hague, Netherlands. Am J Clin Nutr 2006;84:350-3.

18. Hollis BW, Wagner CL. Vitamin D and pregnancy: skeletal effects, nonskeletal effects, and birth outcomes. Calcif Tissue Int 2013;92:128-39.

19. Aghajafari F, Nagulesapillai T, Ronksley PE, et al. Association between maternal serum 25-hydroxyvitamin $D$ level and pregnancy and neonatal outcomes: systematic review and meta-analysis of observational studies. BMJ 2013;346.

20. Poel YH, Hummel P, Lips $\mathrm{P}$, et al. Vitamin D and gestational diabetes: a systematic review and meta-analysis. Eur J Intern Med 2012;23:465-9.

21. Wei SQ, Qi HP, Luo ZC, et al. Maternal vitamin D status and adverse pregnancy outcomes: a systematic review and meta-analysis. J Matern Fetal Neonatal Med 2013;26:889-99.

22. Tabesh M, Salehi-Abargouei A, Tabesh M, et al. Maternal vitamin D status and risk of pre-eclampsia: a systematic review and meta-analysis. J Clin Endocrinol Metab 2013:98:3165-73.

23. Thorne-Lyman A, Fawzi WW. Vitamin D during pregnancy and maternal, neonatal and infant health outcomes: a systematic review and meta-analysis. Paediatr Perinat Epidemiol 2012;26(Suppl 1):75-90.

24. Javaid MK, Crozier SR, Harvey NC, et al. Maternal vitamin D status during pregnancy and childhood bone mass at age 9 years: a longitudinal study. Lancet 2006;367:36-43.

25. Harvey NC, Moon RJ, Sayer AA, et al, Southampton Women's Survey Study Group. Maternal antenatal vitamin D status and offspring muscle development: findings from the Southampton Women's Survey. J Clin Endocrinol Metab 2013;99:330-7.

26. Martin R, Harvey NC, Crozier SR, et al. Placental calcium transporter (PMCA3) gene expression predicts intrauterine bone mineral accrual. Bone 2007:40:1203-8.

27. Cleal JK, Day PE, Simner CL, et al. Placental amino acid transport may be regulated by maternal vitamin $D$ and vitamin $D$-binding protein: results from the Southampton Women's Survey. Br J Nutr 2015;113:1903-10.

28. Javaid MK, Lekamwasam S, Clark J, et al. Infant growth influences proximal femoral geometry in adulthood. J Bone Miner Res 2006;21:508-12.
29. Ross AC. The 2011 report on dietary reference intakes for calcium and vitamin D. Public Health Nutr 2011;14:938-9.

30. Holick MF, Binkley NC, Bischoff-Ferrari HA, et al. Evaluation, treatment, and prevention of vitamin D deficiency: an Endocrine Society clinical practice guideline. J Clin Endocrinol Metab 2011;96:1911-30

31. ACOG Committee on Obstetric Practice. ACOG Committee Opinion No. 495: vitamin D: screening and supplementation during pregnancy. Obstet Gynecol 2011;118:197.

32. WHO Guideline. Vitamin D supplementation in pregnant women. 2012. http://apps.who.int/iris/bitstream/10665/85313/1/ 9789241504935_eng.pdf?ua $=1$ (accessed Nov 2015).

33. De-Regil LM, Palacios C, Ansary A, et al. Vitamin D supplementation for women during pregnancy. Cochrane Database Syst Rev 2012;2:CD008873.

34. NICE Guidelines. 2008. http://www.nice.org.uk/guidance/PH11 (accessed Nov 2015)

35. Hollis BW, Johnson D, Hulsey TC, et al. Vitamin D supplementation during pregnancy: double-blind, randomized clinical trial of safety and effectiveness. J Bone Miner Res 2011;26:2341-57.

36. Cooper C, Harvey N, Bishop N, et al, MAVIDOS Study Group. Maternal Gestational Vitamin D Supplementation and Offspring Bone Mass: a Multicentre Randomised, Double-Blind, Placebo-Controlled Trial (MAVIDOS). Abstract Presented at the American Society for Bone and Mineral Research (ASBMR) Annual Meeting; 9-12 October 2015; Seattle; 2015.

37. Sablok A, Batra A, Thariani K, et al. Supplementation of vitamin D in pregnancy and its correlation with feto-maternal outcome. Clin Endocrinol (Oxf) 2015;83:536-41.

38. Dawodu A, Saadi HF, Bekdache G, et al. Randomized controlled trial (RCT) of vitamin $\mathrm{D}$ supplementation in pregnancy in a population with endemic vitamin d deficiency. J Clin Endocrinol Metab 2013;98:2337-46.

39. Sabet Z, Ghazi A, Tohidi M, et al. Vitamin D supplementation in pregnant Iranian women: effects on maternal and neonatal vitamin D and parathyroid hormone status. Acta Endocrinol 2012:8:59-66.

40. Soheilykhah S, Mojibian M, Moghadam MJ, et al. The effect of different doses of vitamin D supplementation on insulin resistance during pregnancy. Gynecol Endocrinol 2013;29:396-9.

41. Shakiba M, Iranmanesh MR. Vitamin D requirement in pregnancy to prevent deficiency in neonates: a randomised trial. Singapore Med $J$ 2013;54:285-8.

42. ClnicalTrials.Gov. https://clinicaltrials.gov/ (accessed Nov 2015).

43. College of American Pathologists. http://www.cap.org (accessed Nov 2015).

44. DEQAS. http://www.deqas.org (accessed Nov 2015)

45. World Bank definition of Middle East countries. http://data worldbank.org/about/country-and-lending-groups (accessed Nov 2015).

46. Skinner AM, Cieslak Z, MacWilliam L, et al. The measurement of knee-heel length in newborn infants using a simple vernier calipers. Acta Paediatr 1997;86:512-17.

47. Bender R, Lange S. Adjusting for multiple testing-when and how? $J$ Clin Epidemiol 2001;54:343-9.

48. Online sample size calculation. http://www.stat.ubc.ca/ rollin/stats/ ssize/ (accessed Nov 2015).

49. Heaney RP, Davies KM, Chen TC, et al. Human serum 25-hydroxycholecalciferol response to extended oral dosing with cholecalciferol. Am J Clin Nutr 2003;77:204-10.

50. Little RJ, D'Agostino R, Cohen ML, et al. The prevention and treatment of missing data in clinical trials. $N$ Engl $\mathrm{J} \mathrm{Med}$ 2012;367:1355-60.

51. Duke Radition Safety. http://www.safety.duke.edu/Radsafety/ consents/default.asp (accessed Nov 2015).

52. Heaney RP. Guidelines for optimizing design and analysis of clinical studies of nutrient effects. Nutr Rev 2014;72:48-54. 